\title{
The effect of chronic intoxication by organophosphate insecticides on the parameters of innate and adaptive immunity and realization of the cholinergic anti-inflammatory pathway
}

\begin{abstract}
Experiments on noninbred albino rats showed that chronic intoxication with organophosphorus compounds (organophosphorus insecticides) malathion and parathion methyl $\left(0.01 L_{50}\right.$ daily, for 30 days) significantly reduces the phagocytic-metabolic activity of neutrophils, the activity of natural killer, antibody-dependent cellular cytotoxicity and the production of proinflammatory cytokines TNF $\alpha$, IL-I $\beta$ and IL-6. The chronic organophosphorus insecticides intoxication leads to the realization of the cholinergic antiinflammatory pathway.
\end{abstract}

Keywords: organophosphate insecticides, neutrophil, immunity, cholinergic antiinflammatory pathway, cytokines
Volume 6 Issue 6 - 2018

\section{Pavel Franzevich Zabrodskii}

Saratov Medical University “REAVIZ”, Russia

Correspondence: Pavel Franzevich Zabrodskii, Saratov Medical University "REAVIZ", st. Maxim Gorky, house 13, apartment. 66, 410028, Russia, Tel +7 90532327 5I, Email pfzabradsky@gmail.com

Received: October 30, 2018 | Published: November 12, 2018

\section{Introduction}

A large number of anticholinesterase substances are used in agriculture, in various branches of industry, in medicine and in everyday life. Basically, these are substances related to organophosphate insecticides (organophosphorus compounds-OPC). The widespread use of these toxicants as insecticides, as well as the destruction of combat OPC, can lead to environmental pollution, causing intoxication of people and animals. ${ }^{1-3}$ There is no doubt that research into the mechanisms of the formation of a post intoxication immunodeficiency state in the poisoning of OPC, in particular, the function of phagocyticmonocyte system (PMS) for the purpose of prevention and treatment, resulting in various infectious complications and diseases. ${ }^{2,3}$ With the chronic effect of the OPC, the implementation of the cholinergic antiinflammatory pathway (mechanism) ${ }^{4-13}$ which includes: activation of $\mathrm{m}$-cholinergic receptors ( $\mathrm{mAChRs}$ ) of the brain, which modulate the immunoregulatory function of the vagus nerve; excitation of efferent fibers $n$. vagus; the action of acetylcholine on receptors; the activation of $n$-cholinergic receptors (in particular, $\alpha 7 \mathrm{nAChRs)} \mathrm{of} \mathrm{PMS} \mathrm{cells.}$ In PMS cells, the onset of an anti-inflammatory effect is provided by kinase JAK2; transcription factor STAT3; transcription factor NF$\kappa \mathrm{B}$. Under the influence of cholinergic stimulation, the "inclusion" of these biochemical mechanisms of the cell inhibits the production of TNF- $\alpha$, B1-HMGB1 protein, macrophage-inflammatory protein-2MIP-2, interleukins IL-1 $\beta$, IL-6..$^{10,11,13,14}$ Thus, cholinergic stimulation causes acetylcholine $\alpha 7 \mathrm{nAChRs}$ activation of the PMS system cells (macrophages, monocytes and neutrophils), which leads to a decrease in the production of these cells (as well as lymphoid dendritic cells) of proinflammatory cytokines. . $^{2,37-10,15,16}$ The effect of chronic organophosphate insecticides intoxication (malathion and parathion methyl) on neutrophil function and the implementation of the cholinergic anti-inflammatory pathway has not been adequately investigated.

\section{Aim of the study}

The aim of the study was to determine the effect of chronic intoxication of organophosphate insecticides on the phagocyticmetabolic activity of neutrophils, function cells of the immune system, the blood levels of the pro-inflammatory cytokines TNF $\alpha$, IL- $1 \beta$ and IL- 6 produced by them, and realization of the cholinergic anti-inflammatory pathway.

\section{Materials and methods}

The experiments were carried out on random-bred albino rats of both sexes weighing $18-22 \mathrm{~g}$. The control group of rats received isotonic sodium chloride solution (saline) within 30days corresponding to the volume of aqueous solution of an OPC emulsion in groups of rats treated with OPC $(0.5-1.0 \mathrm{ml})$. The OPC (malathion-MT-[O,ODimethyl-S-(1,2-dicarbethoxyethyl) dithiophosphate] and parathion methyl-PM-[O,O-diethyl O-(4-nitrophenyl) phosphorothioate $]$ ) were injected subcutaneously daily for 30 days at a dose of $0.01 \mathrm{LD}_{50}$ $\left(\mathrm{LD}_{50}\right.$ of these insecticides were injected subcutaneously with $815 \pm 28$ (MT) and $25.3 \pm 2.6 \mathrm{mg} / \mathrm{kg}(\mathrm{PM})$. Used the aqueous solutions of $50 \%$ MT emulsion ("AgroWista", Russia) and PM ("AgroVista", Russia) The phagocytic-metabolic activity of neutrophils was assessed by 30 days after the first dose of OPC was administered by determining the phagocytic index, phagocytic number, neutrophil activity index (NAI) in spontaneous and induced Nitro Blue-Tetrazolium Test (NBT, NBT spontaneous, NBT induced) generally accepted methods. S. aureus 209P was used to assess the phagocytic index and the phagocytic number. ${ }^{2,3,17,18}$

Parameters of cellular immune reactions were assessed by generally accepted methods in experimental immunotoxicology and immunology $2^{2,3,19}$ after chronic intoxication of OPC 30 days after the first injection of organophosphorous substances. The activity of natural killer (NK) was determined by the index of natural cytotoxicity spectrophotometrically. Antibody-dependent cellular cytotoxicity (ADCC) was determined on 4 days after immunization of rats of sheep red blood cells $\left(10^{8}\right)$, using their splenocytes spectrophotometrically. ${ }^{2,3}$ Immunization was performed on 26 days after the first injection of OPC. When evaluating phagocytic-metabolic activity of neutrophils 
and immune responses, animals received a total dose of OPC (MT and PM), which is $0.3 \mathrm{LD}_{50}$. The concentration of TNF- $\alpha$, IL1 $\beta$ and IL-6 was determined in blood plasma of rats after chronic intoxication OPC by enzyme immunosorbent assay (ELISA) using kits (ELISA Kits MyBioSoure) in accordance with the manufacturer's instructions. Monoclonal antibodies MyBioSoure (TNF- $\alpha$, IL1 $\beta$, IL-6\#MBS494184, \#MBS494492, \#MBS335516) were used to determine the concentration of pro-inflammatory cytokines. Blood for research was taken from the retroorbital venous sinus. The data obtained were processed statistically using the Student's t-test. Differences between the parameters were considered reliable at $\mathrm{p}<0.05$.

\section{Results}

Under the influence of chronic intoxication of OPC a significant reduction of phagocytic-metabolic activity of neutrophils was noted
(Table 1). 30 days after the action of MT the phagocytic index, phagocytic number, neutrophil activity index in NBT spontaneous, NBT induced decreased, respectively, to $1.77 ; 1.49 ; 1.94$ and 1.24 times $(\mathrm{p}<0.05)$, and after intoxication with PM-1.48; $1.66 ; 1.55$ and 1.33 times $(\mathrm{p}<0.05)$, respectively. The activity of NK and ADCC under the influence of MT decreased, respectively, 1.30 and 1.46 times ( $\mathrm{p}<0.05$ ), and after chronic intoxication by PM-by 1.55 and 1.32 times $(\mathrm{p}<0.05)$. According to the degree of reduction of phagocyticmetabolic activity of neutrophils, activity of NK and ADCC the effects of OPC in equiletal doses did not differ significantly. The immunosuppressive effect of OPC was accompanied by decrease in the blood concentration of pro-inflammatory cytokines (Table 2). So, after chronic intoxication of MT concentration of TNF $\alpha$, IL-1 $\beta$ and IL-6 in the blood, respectively, decreased by $1.45 ; 1.91$ and 1.55 times $(\mathrm{p}<0.05)$, and after PM intoxication - $1.67 ; 1.63$ and 1.40 times $(\mathrm{p}<0.05)$, respectively.

Table I The changes in the phagocytic-metabolic activity neutrophils and the indices of cellular immune responses of rat under the influence of chronic intoxication of organophosphorus compounds (total dose $0.3 L_{50}, 30$ days) $\left.(M \pm m), n=8-10\right)$

\begin{tabular}{llll}
\hline Parameters & Control group & Malathion & Parathion methyl \\
\hline Phagocytic index, \% & $33,4 \pm 2,9$ & $18,9 \pm 2,3^{*}$ & $22,5 \pm 1,9^{*}$ \\
Phagocytic number, c. u. & $2,54 \pm 0,22$ & $1,70 \pm 0,15^{*}$ & $1,53 \pm 0,17^{*}$ \\
NBT spontaneous, NAI & $0,31 \pm 0,03$ & $0,16 \pm 0,02^{*}$ & $0,20 \pm 0,02^{*}$ \\
NBT induced, NAI & $0,52 \pm 0,05$ & $0,42 \pm 0,04^{*}$ & $0,39 \pm 0,04^{*}$ \\
NK activity, \% & $34,1 \pm 3,0$ & $26,3 \pm 2,3^{*}$ & $22,0 \pm 2,5^{*}$ \\
ADCC, \% & $13,3 \pm 1,4$ & $9,2 \pm 1,0^{*}$ & $10,1 \pm 1,1^{*}$
\end{tabular}

Note: NAI-neutrophil activity index; c.u.-conventional units (average number of microbial cells absorbed by one neutrophil); ${ }_{-1}<0,05$ as compared to control.

Table 2 The effect of chronic intoxication of organophosphorus compounds (total dose of $0.3 \mathrm{LD}_{50}, 30$ days) on the concentration of pro-inflammatory cytokines in the blood of rats, $\mathrm{pg} / \mathrm{ml}((\mathrm{M} \pm \mathrm{m}, \mathrm{n}=7)$

\begin{tabular}{llll}
\hline Series of experiments & ФНО $\alpha$ & ИЛ І $\boldsymbol{\beta}$ & ИЛ-6 \\
\hline Control group & $87 \pm 9 *$ & $65 \pm 8^{*}$ & $115 \pm 10$ \\
Malathion & $60 \pm 7^{*}$ & $34 \pm 4^{*}$ & $74 \pm 9 *$ \\
Parathion methyl & $52 \pm 6^{*}$ & $40 \pm 5^{*}$ & $82 \pm 8^{*}$ \\
\hline
\end{tabular}

$*_{-p}<0,05$ as compared to control.

\section{Discussion}

It is possible that the reduction of phagocytic-metabolic activity of neutrophils may be associated with inhibition of cytosol esterase by PMS cells $(\alpha$-naphthyl acetate esterase, $\alpha$-naphthyl butyrate esterase, $\alpha$-naphthyl-AS-D-acetasterase, $\quad \alpha$-naphthyl-AS-D-chloroacetate esterase) and also with initiation of OPC by lipid peroxidation. ${ }^{2,3}$ The suppression of the activity of NK and ADCC is probably due to the inactivation of OPC acetylcholinesterase of $\mathrm{NK}$ and cells participating in the ADCC. , $3,20^{2}$ The effect of OPC on phagocytic-metabolic activity of neutrophils is probably due to the interaction of toxicants and their metabolites with NADP.H and $\mathrm{NADH}^{+}$. The action of OPC can also be associated with inhibition by toxicants and products of their biotransformation $\mathrm{FAD}^{+}, \mathrm{FAD} \cdot \mathrm{H}$, reduced and oxidized ubiquinone, cytochrome $\mathrm{b}_{245}$ of leukocytes, or other mechanisms for disrupting the functioning of the NADP.H oxidase complex of neutrophils. OPC also affects oxygen-dependent microbicidal systems of phagocytes (neutrophils) besides to oxygen-dependent anti-infective systems of phagocytosis. ${ }^{2,3,17-19}$
As already mentioned, a decrease in the production of proinflammatory cytokines by neutrophils, monocytes, macrophages (and, to a lesser extent, other cells of the immune system) is due to the cholinergic anti-inflammatory pathway (activated by acetylcholine $\alpha 7 \mathrm{nAChRs}$ cells) under the influence of cholinergic stimulation PMS, leading to a reduction in the synthesis of pro-inflammatory cytokines). ${ }^{2,3,7,8,10,11,15,16}$ Probably a decrease the production of these cytokines during intoxication OPC is not only induced as a result of the mechanisms associated with the cholinergic anti-inflammatory pathway., ${ }^{3,11,21}$ The excitation of neutrophils with acetylcholine $\mathrm{mAChR}$ leads to an increase in their phagocytic-metabolic activity. ${ }^{3,21}$ However, as shown our experiments, with the chronic effect of OPC the opposite effect was observed, due to the action of acetylcholine on $\alpha 7$ nAChRs neutrophils. ${ }^{3,21}$ It is possible that this effect is significantly higher than the activating effect of acetylcholine on the mAChRs of PMS cells.

There is reason to believe that when OPC is intoxicated, the phagocytic-metabolic activity of neutrophils is determined by numerous effects, a number of which are differently directed (for example, activation of neutrophil mAChRs and $\alpha 7 n A C h R s .{ }^{3,21}$ It is interesting to note that a decrease in the synthesis of pro-inflammatory cytokines during cholinergic stimulation, in particular, under the influence of OPC, can lead to a decrease in the mortality of animals in the early stage of sepsis. ${ }^{2-5,9}$ With the defeat of toxicants, in particular, $\mathrm{OPC}$, the phagocytic-monocytic system plays a very important role in the manifestation of both inflammatory and anti-inflammatory effects. The reduction of phagocytic-metabolic activity of neutrophils and the synthesis of pro-inflammatory cytokines can be considered as a negative reaction during the chronic action of OPC. In this case, 
possible violations associated with the destruction and removal of tissues affected by a toxicant, and processes associated with their action..$^{22}$ That is the realization of inflammation, as a reaction that bears a protective-adaptive nature (and not only pathological), is disturbed during chronic intoxication of OPC.

\section{Conclusion}

a. The chronic organophosphorus compounds intoxication (malathion, parathion methyl) for 30 days in a total dose of 0.3 $\mathrm{LD}_{50}$ leads to a decrease of phagocytic-metabolic activity of neutrophils, activity of natural killer and antibody-dependent cellular cytotoxicity.

b. The function of the phagocytic-monocytic system after chronic poisoning of organophosphorus compounds decreases, which is manifested by reduction in the blood concentration of proinflammatory cytokines TNF- $\alpha$, IL- $1 \beta$ and IL- 6 .

c. The chronic intoxication with organophosphate insecticides (organophosphorus compounds) leads to the realization of the cholinergic anti-inflammatory pathway.

\section{Acknowledgements}

None.

\section{Conflict of interest}

Author declares that there is no conflict of interest.

\section{References}

1. Tremolada P, Finizio A, Villa S, et al. Quantitative inter-specific chemical activity in the aquatic environment. Aquat Toxicol. 2004;67(1):87-103.

2. Zabrodskii PF, Mandych VG. Immunotoxicology of xenobiotics. Saratov: Saratov Military Institute of Biological and Chemical Safety; 2007. 420 p.

3. Zabrodskii PF. Immunotoxicology of organophosphorus compounds. Saratov; 2016. $289 \mathrm{p}$

4. Zabrodskii PF. Effect of armin on nonspecific resistance factors of the body and on the primary humoral immune response. Farmakol Toksikol. 1987;50(1):57-60.

5. Zabrodskii PF. Change in the non-specific anti-infection resistance of the body exposed to cholinergic stimulation. Bull Exp Biol Med. 1995;120(8):164-166.

6. Wang $H$, Yu M, Ochani $M$, et al. Nicotinic acetylcholine receptor alpha7 subunit is an essential regulator of inflammation. Nature. 2003;421(6921):384-388.

7. Pavlov VA, Wang H, Czura CJ, et al. The cholinergic anti-inflammatory pathway: a missing link in neuroimmunomodulation. Mol Med. 2003;9(5-8):125-134.

8. Giebeleh IA, Leendertse M, Florquin S, et al. Stimulation of acetylcholine receptors impairs host defence during pneumococcal pneumonia. Eur Respir J. 2009;33(2):375-381.
9. Zabrodskii PF. Effect of acetylcholine on mortality of mice from sepsis and proinflammatory cytokine production. Bull Exp Biol Med. 2011;150(3):340-342.

10. Zabrodskii PF, Gromov MS, Maslyakov VV. Effect of $\alpha 7$ n-Acetylcholine Receptor Activation and Antibodies to TNF- $\alpha$ on Mortality of Mice and Concentration of Proinflammatory Cytokines During Early Stage of Sepsis. Bull Exp Biol Med. 2015;159(6):740-742.

11. Zabrodskii PF, Gromov MS, Maslyakov VV. Role of $\beta 2$-adrenoreceptors in adrenergic anti-Inflammatory mechanism in sepsis. Bull Exp Biol Med. 2016;163(6):710-713.

12. Zabrodskii PF, Gromov MS, Maslyakov VV. Combined Effects of M1 Muscarinic Acetylcholine Receptor Agonist TBPB and $\alpha 7 n$-Acetylcholine Receptor Activator GTS-21 on Mouse Mortality and Blood Concentration of Proinflammatory Cytokines in Sepsis. Bull Exp Biol Med. 2017;162(6):750-753.

13. Zabrodskii PF, Gromov MS, Maslyakov VV. Combined Effect of NF- $\kappa B$ Inhibitor and $\beta 2$-Adrenoreceptor Agonist on Mouse Mortality and Blood Concentration of Proinflammatory Cytokines in Sepsis. Bull Exp Biol Med. 2018;165(4):445-448.

14. Borovikova LV, Ivanova S, Zhang M, et al. Vagus nerve stimulation attenuates the systemic inflammatory response to endotoxin. Nature. 2000;405(6785):458-462.

15. Kessler W, Traeqer T, Westerholt A, et al. The vagal nerve as a link between the nervous and immune system in the instance of polymicrobial sepsis. Langenbecks Arch Surg. 2006;391(2):83-87.

16. Sitapara RA, Antoine DJ, Sharma L, et al. The $\alpha 7$ nicotinic acetylcholine receptor agonist GTS-21 improves bacterial clearance in mice by restoring hyperoxia-compromised macrophage function. $\mathrm{Mol} \mathrm{Med}$. 2014;20:238-247.

17. Kovacs AR, Pal L, Szücs S, et al. Phagocytic function of monocytes and neutrophil granulocytes in ovarian cancer. Orv Hetil. 2018;159(33):1353-1359.

18. Hertiani T, Yuswanto A, Utami Tunjung Pratiwi S, et al. Effect of Massoia (Massoia aromatica Becc.) Bark on the Phagocytic Activity of Wistar Rat Macrophages. Sci Pharm. 2018;86(2):E19.

19. Holsapple MH, Burns-Naas LA, Hastings KL, et al. A proposed testing framework for developmental immunotoxicology (DIT). Toxicol Sci. 2005;83(1):18-24.

20. Hayhoe FGJ, Quaglino D. Haematological Cytochemistry. 3rd ed. Edinburgh, London, New York: Churchill Livingstone; 1994. 673 p.

21. Zabrodskii PF, Lim VG, Shekhter MS, et al. Role of nicotinic and muscular cholinoreceptors during the early phase of sepsis. Bull Exp Biol Med. 2012;153(5):700-703.

22. Laskin DL, Sunil VR, Gardner CR, et al. Macrophages and tissue injury: agents of defense or destruction?. Annu Rev Pharmacol Toxicol. 2011;51:267-288. 